\title{
LES DERNIERS FRANÇAIS DE FRENCHVILLE
}

\author{
par Françoise MougeON et Dorin URITESCU \\ Université York, Collège universitaire Glendon
}

This article proposes a systematic study of the most salient linguistic features of the French spoken in Frenchville, Pennsylvania, at the end of the XXth century. It also attempts to account for their origin and explanation in relation with the wolution of popular varieties of French and of northern Gallo-Roman dialects, and in the sociolinguistic context of North America.

Dans le cadre d'une étude d'un corpus d'entrevues de deux octogénaires de Frenchville enregistrées en 1988 dans cette localité ${ }^{1}$, nous analyserons plus spécifiquement ici les phénomènes suivants: l'effacement du /l/ dans les pronoms clitiques sujets, l'emploi du pronom eux en position sujet, l'emploi de l'auxiliaire avoir dans les temps composés du passé, le traitement du pronom relatif et un aspect de la morphosyntaxe du subjonctif, phénomènes résultant de l'évolution de cette variété de français, et les cas d'alternance de code liés au contact de l'anglais.

\section{L'EFFACEMENT DU /L/ \\ DANS LES PRONOMS PERSONNELS SUJETS}

Pour étudier le rapport entre le français de nos locuteurs, le français populaire et les dialectes d'origine, nous avons analysé un phénomène connu dans la plupart des variétés du français et des dialectes d'oïl : l'effacement du /l/ dans les pronoms clitiques sujets : $i$ vient, $\varepsilon / a$ vient, etc.

1. Ce corpus a été constitué par Édouard Beniak. Nous le remercions de nous avoir donné accès à ses données. 
Historiquement, il s'agit d'un processus phonologique, notamment la vocalisation et l'amuïssement du /l/ suivi d'une consonne: lat. filicella $>$ fr. ficelle. En ancien français (déjà au $\mathrm{XII}^{\mathrm{c}}$ siècle) le processus s'étend phonétiquement au /l/ du pronom il (sg. et pl.) suivi d'un verbe commençant par une consonne, ce qui suggère que la liaison entre le pronom sujet et le verbe est devenue plus étroite. Dans ce contexte, le processus phonologique est morphologisé, c'est-à-dire qu'il acquiert un conditionnement morphologique, et, après l'amüssement du schwa de la syllabe finale, s'étend au pronom sujet elle, en tant que processus à double conditionnement, à la fois phonologique et morphologique (que nous appellerons processus «morphophonologique $")^{2}$.

La généralisation morphophonologique est déjà amorcée en français populaire au XVII ${ }^{e}$ siècle. Les grammairiens de l'époque remarquent l'effacement du /l/ dans le pronom elle quand le mot suivant commence par une consonne. Bérain note le phénomène en 1675 : «Plusieurs en parlant de filles ou de femmes disent : il faut qu'alle donne, alle est, a n'est pas. $"^{3}$

Il semble même que le patois parisien soit allé plus loin. En effet, dans les Agréables conférences... on trouve un système qui semble avoir commencé la généralisation de la forme /i/ pour il devant une voyelle :

a) il : $i$ devant consonne (et y di sdity... " et il dit, se dit-il... "); il devant une voyelle $(y$ lest), mais aussi $i$ (quer y a Guieu et rairon de son couté « car il a Dieu et raison..." $)^{4}$.

b) elle: al (alle) devant voyelle (a lavet); al et $a$ devant consonne (al fy tan...; mais : queme y vire qua fezet la defficible $)^{5}$.

2. Pour les détails de ces changements historiques, ainsi que pour la distinction entre les processus phonologiques et les processus morphophonologiques (ou à double conditionnement, phonologique et morphologique), voir Dorin Uritescu, 1997, « L'effacement du /1/ en québécois et le type morphologique du français", Revue de linguistique romane, t. 61, $\mathrm{n}^{\infty} 243-244$, p. $397-437$

3. Apud Charles Thurot, 1966, De la prononciation frangaise: depuis le commencement du $X V T$ siècle, d'après les témoignages des grammainiens, Genève, Slatkine Reprints, I, p. 22.

4. Frédéric Deloffre, 1961, Agréables conférences de deux paysans de Saint-Ouen et de Montmorency sur les affaires du temps (1649-1651), édition critique par Frédéric Deloffre, Paris, Société d'édition "Les Belles Lettres ", p. 43/63, 40/26, 49/120 ; Théodore Rosset, 1911, Les origines de la prononciation modeme étudiées au XVIr siècle d'après les remarques des grammainiens et les textes en patois de la banlieue parisienne, Paris, Colin, p. 6/6.

5. Deloffre, Agréables conferences..., p. 165; cf. aussi Rosset, Les ongines..., p. 393. 
Le processus morphophonologique est donc déjà en train de se transformer en processus allomorphique (sans conditionnement phonologique). Ce système est plus " avancé " que le système de beaucoup de variétés gallo-romanes contemporaines.

En effet, dans beaucoup de ces variétés, /i/ est fréquent devant les verbes qui commencent par une consonne. L'effacement du /l/ dans ce contexte a été étendu au féminin dans la plupart des parlers de la région briarde et de la région parisienne ${ }^{6}$, ainsi que dans beaucoup d'autres parlers populaires de France ${ }^{7}$.

La tendance à généraliser l'effacement du /l/ devant une voyelle dans le pronom il (et même dans le pronom elle) se retrouve pourtant en français populaire ${ }^{8}$.

Voyons maintenant quelle est la situation dans deux des principales régions d'origine de nos locuteurs: la Haute-Marne et la Haute-Saône. Si on laisse de côté la forme il y a, dans laquelle l'effacement du /1/ peut être phonétique (comme le prouvent les formes régionales avec [n]), le processus ressemble partiellement à celui du patois de la région parisienne au $\mathrm{XVII}^{e}$ siècle : pour $i l:[\mathrm{i}] /[\varepsilon]$ devant une consonne, $[\mathrm{il}] /[\varepsilon]$ et rarement $[\mathrm{i}] /[\varepsilon]$ devant une voyelle (dans trois points de la Haute-Marne). Il y a pourtant une grosse différence pour le féminin elle : [ $[$ ] partout (dans quelques points [al] ou $[ø]])$, donc devant une voyelle et devant une consonne ${ }^{9}$.

La spécificité des deux régions (en fait, la Haute-Saône et trois points en Haute-Marne) est établie de façon claire par Colette Dondaine ${ }^{10}$ :

- il: [è]/[a] devant consonne; [èl]/[al] devant voyelle ;

- ils : [è] devant consonne ; [èl] devant voyelle ;

- elle: [èl] devant consonne ; [èll] (/ [èl] dans un point) devant voyelle ;

- elles: [èl] devant consonne; [èll] devant voyelle.

6. Marguerite Durand, 1936, Le genre grammatical en frangais parlè à Paris et dans la région parisienne, Paris, Bibliothèque du français moderne, p. 172-175, etc. 4, 5; Yves-Charles Morin, 1979, "La morphophonologie des pronoms clitiques en français populaire ", Cahiers de linguistique, 9, p. 1-36.

7. Jules Gilliéron et Edmond Edmont, 1902-1910, Atlas linguistique de la France, Paris, Champion, c. $1283,1251,1404$.

8. Voir William Ashby, 1984, «The elision of $/ /$ in French clitic pronouns and articles ", in Ernst Pulgram (dir.), Romanitas: Studies in Romance Linguistics, Ann Arbor, Michigan Romance Studies, p. 1-16.

9. Gilliéron et Edmont, $A L F$, c. 95, 103, 462, 498 b, 502, 509, 510, 674, 1035, $1036 b, 1404$.

10. Colette Dondaine, 1991, Atlas linguistique et ethnographique de la Frenche-Comté, IV, Paris, CNRS, c. 1247-1254. 
Nous analyserons maintenant le système de nos locuteurs de Frenchville $^{11}$ :

Locuteur I (EV)

\begin{tabular}{|c|c|c|c|c|c|c|c|c|c|c|c|c|}
\hline & \multicolumn{4}{|c|}{ Il impersonnel } & \multicolumn{4}{|c|}{ Il personnel } & \multicolumn{4}{|c|}{ Elle } \\
\hline $\begin{array}{l}\text { Total } \\
\text { occ. }\end{array}$ & \multicolumn{4}{|c|}{73} & \multicolumn{4}{|c|}{124} & \multicolumn{4}{|c|}{97} \\
\hline & \multicolumn{2}{|c|}{$+\mathrm{V}$} & \multicolumn{2}{|c|}{$+\mathrm{C}$} & \multicolumn{2}{|c|}{$+\mathrm{V}$} & \multicolumn{2}{|c|}{$+\mathrm{C}$} & \multicolumn{2}{|c|}{$+\mathrm{V}$} & \multicolumn{2}{|c|}{$+\mathrm{C}$} \\
\hline & L & 0 & L & 0 & L & 0 & L & 0 & L & 0 & L & 0 \\
\hline$\%$ & 2 & 98 & 0 & 100 & 66 & 34 & 2 & 98 & 100 & 0 & 67 & 33 \\
\hline
\end{tabular}

Locuteur II (FP)

\begin{tabular}{|c|c|c|c|c|c|c|c|c|c|c|c|c|}
\hline & \multicolumn{4}{|c|}{ Il impersonnel } & \multicolumn{4}{|c|}{$I l$ personnel } & \multicolumn{4}{|c|}{ Elle } \\
\hline $\begin{array}{l}\text { Total } \\
\text { occ. }\end{array}$ & \multicolumn{4}{|c|}{12} & \multicolumn{4}{|c|}{16} & \multicolumn{4}{|c|}{23} \\
\hline & \multicolumn{2}{|c|}{$+\mathrm{V}$} & \multicolumn{2}{|c|}{$+\mathrm{C}$} & \multicolumn{2}{|c|}{$+\mathrm{V}$} & \multicolumn{2}{|c|}{$+\mathrm{C}$} & \multicolumn{2}{|c|}{$+\mathrm{V}$} & \multicolumn{2}{|c|}{$+\mathrm{C}$} \\
\hline & L & 0 & L & 0 & L & 0 & L & 0 & L & 0 & L & 0 \\
\hline$\%$ & 0 & 100 & 0 & 100 & 88 & 12 & 0 & 100 & 100 & 0 & 100 & 0 \\
\hline
\end{tabular}

Comme on peut le voir, le système du locuteur II est plus proche du système dialectal des régions d'origine en ce qui concerne le pronom elle suivi par une consonne. Cela pourrait s'expliquer, à notre avis, par le fait qu'il s'agit d'un locuteur plus restreint, qui aurait donc pu conserver un système plus ancien et plus proche du système dialectal d'origine.

Le système de la locutrice EV (notre locuteur I), d'autre part, n'est pas très différent de celui du français populaire et se caractérise par l'extension de l'effacement du /1/ dans le pronom il au contexte "devant voyelle " et dans le pronom elle au contexte « devant consonne ». On pourrait dire qu'il s'agit d'un processus allomorphique qui n'est que le résultat d'une tendance évolutive du français populaire qui s'annonce déjà au XVII ${ }^{\mathrm{c}}$ siècle.

Notons aussi que ce système est différent de celui d'autres variétés nord-américaines. En effet, le québécois est allé plus loin dans cette direction et semble avoir transformé les formes

11. Pour toute information concernant nos informateurs, voir l'annexe. 
sans /l/ en formes sous-jacentes ${ }^{12}$, tandis que l'acadien conserve toujours le conditionnement phonologique ${ }^{13}$, se limitant donc à un processus morpho-phonologique.

Finalement, nos données sur l'effacement du /l/ pourraient étayer l'hypothèse de Valdman sur l'origine du français de Frenchville et l'explication générale de la plupart des caractéristiques des vernaculaires d'Amérique proposée par Vintilà-Ràdulescu et Carayol et Chaudenson, et adoptée par Valdman ${ }^{14}$. En effet, quant à l'effacement du /l/, le français de Frenchville continue essentiellement le français populaire et son caractère plus "avancé ", notamment la généralisation de l'effacement du /l/ dans le pronom personnel il, pourrait s'expliquer dans une certaine mesure par son isolement par rapport au français standard. Cependant, on retrouve également des traces du système d'origine.

\section{LE PRONOM SUJET [øZ] / [øS]}

Nous n'avons pas analysé chez nos locuteurs le comportement du /1/ dans les formes du pluriel, car celles-ci présentent une caractéristique à Frenchville. Comme on le sait depuis le début des recherches sur cette variété, le français de Frenchville frappe par l'usage d'une forme idiosyncrasique pour le pronom sujet de la troisième personne du pluriel: [øz $]^{15}$. Cette forme n'est pas attestée par les atlas linguistiques dans l'aire d'origine de nos locuteurs, et dans d'autres variétés (par exemple, en québécois), elle apparaît seulement en corrélation avec le singulier lui

12. Uritescu, "L'effacement du /1/... ", p. 404.

13. Ruth King and Terry Nadasdi, 1997, "Left dislocation, number marking and (non-)standard French ", Probus, 9/3, p. 267-284.

14. Albert Valdman, 1980, "L'Ácadie dans la francophonie nord-américaine ", fournal of the Atlantic Provinces Linguistics Association, 2, p. 3-18; Ioana Vintilà-Ràdulescu, 1970, "Français créole et français canadien", Phonétique et linguistique romanes. Mélanges offerts à M. Georges Straka, I, Lyon et Strasbourg, Société de linguistique romane, p. 353-359 ; Michel Carayol et Robert Chaudenson, 1973, "Aperçu sur la situation linguistique de la Réunion ", Cahiers du Centre universitaire de la Réunion, Saint-Denis-de-la-Réunion, Centre universitaire de la Réunion, 3, p. 1-45; cf. aussi Albert Valdman, 1994, "Restructuration, fonds dialectal commun et étiolement linguistique dans les parlers vernaculaires français d'Amérique du Nord ", dans Claude Poirier (dir.), Langue, espace, société, Sainte-Foy, PUL, p. 3-24.

15. Josette Caujolle, 1972, «Esquisse d'une description du parler français de Frenchville ", The French Language in the Americas (Newsletter of the French VIII Section of the $M L A), 16$, p. 26-32 ; Valdman, "L'Acadie... ", p. 10, 13. 
(parle, etc.). Or nos locuteurs n'utilisent jamais la forme tonique de la troisième personne du singulier comme sujet.

Une forme similaire, eusse, est fréquente dans certaines variétés de français de Louisiane ${ }^{16}$. La forme utilisée par nos locuteurs est pourtant distincte de celle utilisée en Louisiane et de celle enregistrée par les autres chercheurs à Frenchville. En effet, chez nos locuteurs, la consonne [z] s'assimile à la consonne initiale du verbe. On a donc deux variantes morphophonologiques:

- [øz] devant une voyelle ou consonne sonore : [øz] veulent, vendaient, ont, etc. ;

- [øs] devant une consonne non voisée : [øs] parlaient, fumaient, peuvent, etc.

Cette tendance à l'assimilation, explicable certainement par un processus phonétique naturel, semble assez récente, car nous avons enregistré aussi [øz] portaient (deux fois), ainsi qu'une forme " hypercorrecte ", [øs] disaient (qui pourrait pourtant être interprétée comme [øs] se disaient).

La forme [øz]/[øs] est toujours majoritaire chez EV (locuteur I ; 36 sur 58 occurrences du pluriel, soit $62 \%$ ), qui l'emploie une fois pour le pluriel féminin (il n'y a que trois pluriels féminins et la locutrice utilise une fois [øz] : [øz] marchaient pour aller nettoyer à l'église, et deux fois $[\varepsilon z]+$ voyelle : [ $[z]$ étaient).

Chez le locuteur II, [øz] est minoritaire, 38,46\% des occurrences du pluriel masculin (le seul pluriel enregistré chez ce locuteur), mais le nombre d'occurrences du pluriel se limite à 13. Il est pourtant à remarquer que chez ce locuteur [øz] n'apparaît que devant une voyelle, à côté de [iz] : [øz] ont (quatre fois) et [øz] avaient, mais aussi [iz] ont (quatre fois). Rappelons qu'il s'agit aussi d'un locuteur plus restreint, qui garde également la distinction morphophonologique entre il et elle.

Contrairement aux apparences, l'explication de la forme $[ø z] /[ø s]$ dans la variété de Frenchville n'est pas simple. Comme nous l'avons dit plus haut, dans cette variété elle n'est pas en relation avec l'emploi de lui comme sujet (comme en québécois), un emploi qui pourrait faire penser à des formes du type lui, il parle, eux, ils parlent, etc., avec diverses variantes du il, ils, etc.

16. Kevin J. Rottet, 2001, Language Shift in the Coastal Marshes of Louisiana, New York, Peter Lang, p. 207. 
Ces constructions ont certainement joué un rôle dans l'apparition de $[ø z] /[ø s]$, mais, à notre avis, d'autres facteurs ont probablement contribué à l'émergence de l'innovation: les anomalies du pronom de la troisième personne dans les dialectes d'origine de nos locuteurs, surtout le manque de distinction entre le singulier et le pluriel dans tous les contextes et le manque de distinction de genre, qui n'est marqué que par un processus morphophonologique en train de s'affaiblir (voir supra); un possible croisement entre la forme dialectale [øl] (pour elle; voir supra) et les formes du français populaire [iz] et $[\varepsilon z]^{17}$.

Finalement, il faut noter que les autres pluriels présentent beaucoup de variations, les formes anciennes côtoyant les formes les plus récentes: [i] + consonne ou voyelle, [il] plus voyelle et même consonne ([il] veulent), [iz] ou [zz] devant une voyelle.

\section{UTILISATION DES AUXILIAIRES AVOIR ET ÊTRE}

Caujolle avait déjà observé la quasi-absence de l'auxiliaire être dans les temps verbaux composés ${ }^{18}$. Nos données indiquent en outre que l'usage de avoir est généralisé même aux verbes pronominaux. Mougeon et al. avait en effet identifié dans les communautés franco-ontariennes, selon le taux de maintien du français, un continuum dans la non-utilisation de cet auxiliaire au profit de l'auxiliaire avoir surtout avec les verbes de mouvement. La généralisation de l'auxiliaire avoir a aussi été relevée dans d'autres variétés de français ${ }^{19}$. Gadet avait également relevé la substitution de avoir à être dans de nombreux cas où la variété standard demande l'utilisation de être dans les temps composés ${ }^{20}$.

17. Une forme /ol/ est attestée dans un autre isolat d'Amérique du Nord; Valdman, «L'Acadie... ", p. 13. Valdman, "Restructuration.... ", p. 16-17, parle aussi d'une tendance à la réduction des formes pronominales dans les vernaculaires d'Amérique du Nord.

18. Caujolle, "Esquisse... ", p. 31 .

19. Michael Canale, Raymond Mougeon et Monique Bélanger, 1978, "Analogical Leveling of the auxiliary être in Ontarian French n, dans S. Suñer (dir.), Contemporary Studies in Romance Linguistics, Washington DC, Georgetown University Press, p. 41-61 ; Gillian Sankoff et Pierrette Thibault, 1980, "The alternation between the auxiliaries avoir and être in Montréal French ", dans Gillian Sankoff (dir.), The Social Life of Language, Philadelphia, University of Pennsylvania Press, p. 311-345 ; Marijke Russo et Julie Roberts, 1999, "Linguistic change in endangered dialects: The case of alternation between avoir and être in Vermont French ", dans Language variation and Change, 11, p. 67-86.

20. Françoise Gadet, 1992, Le frangais populaire, Paris, PUF, p. 55. 
Utilisation de avoir et être par les deux locuteurs

\begin{tabular}{|r|r|c|c|r|c|c|c|c|c|c|c|c|c|}
\hline & \multicolumn{4}{|c|}{ AVOIR } & \multicolumn{3}{c|}{0 aux } & \multicolumn{5}{|c|}{ E.TRE } & T. \\
\hline pr. & dép. & mouv. & autres & T. & & pr. & dép. & mouv. & $\begin{array}{c}\text { naître } \\
\text { mourir }\end{array}$ & autres & T. & \\
\hline EV & $\mathbf{1 2}$ & $\mathbf{0}$ & $\mathbf{2 6}$ & $\mathbf{2 1 0}$ & $\mathbf{2 4 8}$ & 0 & $\mathbf{0}$ & $\mathbf{5}$ & $\mathbf{1}$ & $\mathbf{1 3}$ & $\mathbf{1}$ & 20 & 268 \\
\hline FP & $\mathbf{6}$ & $\mathbf{4}$ & $\mathbf{2 0}$ & $\mathbf{5 6}$ & $\mathbf{8 2}$ & 4 & $\mathbf{0}$ & $\mathbf{0}$ & $\mathbf{0}$ & $\mathbf{0}$ & $\mathbf{0}$ & 0 & 86 \\
\hline$\%$ & $\mathbf{1 0 0}$ & $\mathbf{4 4 , 4}$ & $\mathbf{9 7 , 8}$ & $\mathbf{9 6}$ & 93,2 & $\mathbf{1 , 1 2}$ & $\mathbf{0}$ & $\mathbf{5 5 , 5}$ & $\mathbf{2 , 1 2}$ & $\mathbf{8 6 , 6}$ & $\mathbf{0 , 3}$ & 5,6 & \\
\hline
\end{tabular}

Légende : pr. : verbes pronominaux ; dép. : verbes dépronominalisés ; mouv. : verbes de mouvement ; T. : total ; EV et FP : locuteurs.

L'utilisation massive de l'auxiliaire avoir prévaut chez nos deux locuteurs. Cependant on peut noter les différences suivantes entre les deux :

- L'absence totale d'utilisation de être pour FP alors que cet auxiliaire est connu et utilisé par EV, quelquefois en distribution parallèle pour les verbes naître, mourir, venir, se marier : quand cte missionnaire là est venu / on a venu; ... que sont mariées / elle s'a remariée, quand j'm'ai mariée; qu'a né ici / ma mère est née.

Comme en français populaire ${ }^{21}$, l'alternance entre les deux auxiliaires semble correspondre à une distinction sémantique entre processus (avoir) et résultat (être).

- L'auxiliaire être n'est jamais utilisé avec des verbes pronominaux. Pour EV il semble que l'auxiliaire être s'accompagne d'une dépronominalisation des verbes pronominaux en français standard: Que sont mariés (qui se sont mariés); Ze suis marié (je me suis mariée).

Il se peut donc que le français de Frenchville continue une variété dialectale ou populaire dans laquelle l'auxiliaire être n'a jamais été généralisé aux verbes pronominaux. On sait que beaucoup de chercheurs ont établi que, dans l'ensemble de la Romania, l'auxiliaire être est apparu tout d'abord avec les verbes non accusatifs et s'est généralisé plus tard par le biais des verbes pronominaux passifs aux verbes pronominaux réfléchis. Cette variété se rattacherait donc à d'autres variétés populaires de France ou d'Italie $^{22}$. Par ailleurs, Gadet constate aussi que la généralisation de avoir est plus fréquente dans les verbes pronominaux ${ }^{23}$.

21. Ibid., p. 55 . p. $19-23$.

22. Rebecca Posner, 1996, The Romance languages, Cambridge, University Press,

23. Gadet, Le frangais..., p. 55. 
EV utilise toujours un auxiliaire pour marquer le temps composé. FP, par contre, utilise parfois une forme du temps composé réduite au participe passé : après f̧a les Russiens venus sauter su'l'dos

Il ressort de la première observation que EV semble avoir été exposée à l'utilisation (même minimale) de formes verbales composées avec être, formes qu'elle s'est appropriées puisque l'on en retrouve quelques occurrences dans sa production. On a même trouvé un exemple d'utilisation de l'auxiliaire être dans un cas où le français standard demanderait l'utilisation de avoir Ge suis pas manqué un jour). Peut-être est-ce là une tendance à généraliser la distinction sémantique mentionnée plus haut (si le sens de cet exemple est «je n'ai pas été absente un seul jour» $)^{24}$. Cependant l'utilisation qu'elle fait de cet auxiliaire demeure restreinte à de rares verbes.

$\mathrm{FP}$, au contraire, ne montre aucune reconnaissance de l'existence de l'auxiliaire être, ce qui porterait à penser que cet auxiliaire ne fait pas partie de sa compétence active ou même passive.

L'utilisation de ces deux auxiliaires varie donc (quoique faiblement) chez nos deux locuteurs, et la suprématie de avoir semble aussi varier avec la restriction (FP étant le locuteur le plus restreint). La quasi-disparition de cet auxiliaire pourrait dans ce cas indiquer une simplification du système morphologique verbal plus avancée que dans d'autres variétés de français.

\section{RÉsultat DU CONTACT DES LANGUES}

Le fait qu'aucune scolarisation en français n'ait été accessible aux habitants de Frenchville et à leurs descendants après l'arrêt de l'immigration aux environs de 1870, a créé les conditions idéales pour faciliter l'influence de l'anglais, langue majoritaire en Pennsylvanie, sur le français des habitants de Frenchville. Aucun autre contact avec le français d'autres régions d'Amérique n'ayant eu lieu, et les contacts avec d'autres populations francophones s'étant limités à de rares voyages en Europe des habitants de Frenchville, le vocabulaire et la grammaire du parler local n'ont pas été affectés par d'autres variétés de français.

L'influence de l'anglais est donc massive et se traduit par des phénomènes d'alternance de code et par de nombreux calques de

24. Cf. il est bu, en face de il a bu, en français populaire ; Gadet, Le franģais..., p. 55. 
l'anglais. Par alternance de code, nous entendons le passage du français à l'anglais quelle que soit la durée des parties empruntées. On comprendra donc par alternance de code les cas d'insertion lexicale simple, l'alternance intraphrastique et l'alternance interphrastique.

Si nos deux locuteurs font un usage abondant de l'alternance de code, ils ne le font pas de la même façon. Nous avons examiné leur comportement en ce qui concerne l'alternance intraphrastique et l'alternance interphrastique.

Ex. Intra- : Le T est SILENT; Une RUN un STORE à BEECH CREEK.

Ex. Inter-: OH, ITS BETTER FOR YOU, WELL, I COME UP LN SIXTY-THREE, y a vingt-cinq ans.

Dans le premier exemple, l'idée de l'énoncé est exprimée dans les deux langues alternativement, alors que dans le deuxième exemple, chaque idée, correspondant à un énoncé distinct, est exprimée dans une langue unique et le passage d'une langue à l'autre signale un changement d'idée et d'énoncé.

Dans la catégorie des cas d'alternance intraphrastique, nous avons inclus le cas particulier de l'insertion lexicale: J'ai eu une HEARING.

Nous avons examiné la proportion d'énoncés correspondant à ces deux cas de figure chez nos deux locuteurs.

Alternance intra- et interphrastique chez les deux locuteurs du corpus

\begin{tabular}{|c|c|c|c|c|c|}
\hline \multicolumn{4}{|c|}{ Alternance intraphrastique } & $\begin{array}{c}\text { Alternance } \\
\text { interphrastique }\end{array}$ & Total \\
\hline & Insertion lexicale & Autres & Total & & \\
\hline EV & $49(71 \%)$ & $20(28,9 \%)$ & $\mathbf{6 9}$ & $12(14,8 \%)$ & $\mathbf{8 1}$ \\
\hline FP & $25(89,2 \%)$ & $2(7,1 \%)$ & $\mathbf{2 8}$ & $15(34,8 \%)$ & $\mathbf{4 3}$ \\
\hline
\end{tabular}

Il ressort du tableau précédent que $\mathrm{EV}$ a produit le double d'occurrences d'alternance de code dans ses énoncés par rapport à FP. Il est bon cependant de remarquer ici l'importance très inégale des deux corpus, le volume d'énoncés produits par EV dépassant de loin celui de FP. On peut donc dire que, proportionnellement, FP a produit plus d'énoncés comportant des alternances de code que EV.

D'autre part, le tableau ci-dessus ne rend pas entièrement compte de la durée des passages alternés. Or nous avons pu 
constater en examinant le corpus que les passages alternés en anglais étaient beaucoup plus longs dans le corpus de FP que dans celui de EV, et que FP alternait toujours du français à l'anglais et revenait rarement au français, à moins qu'un interlocuteur ne prenne l'initiative de lui répondre en français. Nous n'avons pas observé ce comportement chez EV.

Comme on peut le constater, l'importance des insertions lexicales rend compte de la presque totalité des cas d'alternance intraphrastique $(71 \%$ chez EV et $89,2 \%$ chez $\mathrm{FP})$. Ce résultat s'explique en grande partie par le fait qu'il s'agit ici de locuteurs très restreints du français. Contrairement à la plupart des études menées sur l'alternance de code ${ }^{25}$, où les locuteurs étaient des bilingues équilibrés, le présent corpus met en évidence les carences lexicales des locuteurs en français et l'accessibilité des termes anglais qui se substituent aux termes français absents de leur répertoire. On note toutefois une légère différence entre $\mathrm{EV}$ et $\mathrm{FP}$, ce dernier ayant recours plus fréquemment à ce procédé.

Cependant, contrairement à ce qui avait été observé dans l'étude de F. Mougeon, les cas d'alternance intraphrastique ne correspondent pas souvent à une reprise avec reformulation par le biais de l'anglais, suite à une "panne" syntaxique: nous n'avons trouvé qu'un exemple de ce genre: Puis l'autre est... WHERE THE DEVIL IS LIANA?

Cette remarque mise à part, l'alternance de code n'est pas considérée par ces deux locuteurs comme une option, mais est un comportement nécessaire à l'acte de communication ne faisant pas double emploi avec le reste de leur production.

Enfin, FP utilise plus souvent l'alternance entre énoncés complets que EV $(34,8 \%$ pour FP contre $14,8 \%$ pour EV) et, comme nous l'avons mentionné précédemment, EV revient plus volontiers au français que FP après avoir produit un énoncé anglais.

Le comportement de nos locuteurs se démarque donc sensiblement de celui des locuteurs bilingues équilibrés en ce qui

25. David Sankoff, Shana Poplack et S. Vanniarajan, 1991, "The empirical study of code-switching ", dans Papers for the Symposium on Code-Switching in Bilingual Studies: Theory, Significance and Perspectives, Strasbourg, European Science Foundation, p. 181-206; Carol Myers-Scotton, 1998, Codes and Consequences: Choosing Linguistic Varieties, New York, Oxford University Press ; Françoise Mougeon, 1999, Les francophones et leurs styles : variation stylistique dans le frangais parlé de trois locuteurs du Québec, de l'Ontano et de France, thèse de doctorat, Université de Paris X - Nanterre. 
concerne l'alternance de code. Chez les bilingues équilibrés, une telle pratique relève d'une connaissance et d'une conscience poussée des possibilités stylistiques d'un double répertoire linguistique offrant des options diversifiées au locuteur. Chez nos locuteurs, un tel choix n'existe pas ; au contraire, les répertoires linguistiques anglais et français semblent plutôt obéir à une distribution complémentaire et nécessaire.

\section{LE PRONOM RELATIF}

Phénomène déjà observé par d'autres chercheurs, «le langage populaire s'est créé un instrument invariable en généralisant l'usage du pronom relatif que à la place de tous les autres relatifs $~^{26}$. Comme dans d'autres variétés populaires ou régionales de français parlé, le français de nos locuteurs ne comprend qu'un pronom relatif: que. Il remplace tous les pronoms relatifs du français standard: qui, où, que, dont, (dans) lequel, etc.

il y en a en masse de monde que pouvait pas parler frangais; il y avait une de ces scies-là que eux coupaient la glace; je peux prendre un livre qu'il a du frangais dedans...

Les exemples ci-dessus illustrent l'invariabilité de cette « conjonction vide ", dont l'usage comprend un « décumul » laissant apparaître un pronom personnel ${ }^{27}$.

Le locuteur le plus restreint omet fréquemment le pronom relatif (38\% des occurrences), en remplaçant les constructions relatives par des constructions parataxiques:

... je prenais ces petites tasses-là de... STRROFOAM eux appellent fa ; ... passé est un car MODEL T, $i$ m'passe par d'ssus les jambes...

Il s'agit probablement d'un comportement influencé par la restriction linguistique. En effet, EV, locutrice moins restreinte, n'omet pratiquement pas le pronom relatif ( $2 \%$ des occurrences) et cette omission n'est pas accompagnée de l'utilisation de la parataxe.

26. Henri Frei, 1929, La grammaire des fautes, Genève, Bellegarde, SAAGF, p. 182; cf. aussi Henri Bauche, 1951, Le langage populaire, Paris, Payot.

27. Phénomène observé par beaucoup de chercheurs. Par ailleurs, ces constructions, considérées comme plus " transparentes ", se retrouvent en italien populaire et dans beaucoup d'autres langues; $c f$. Anna Giacalone Ramat, 1989, "L'interazione di fattori interni e di fattori esterni nella predicibilità del mutamento linguistico ", dans Vincenzo Orioles (dir.), Modelli esplicativi della diacronia linguistica, Pisa, Giardini editori e stampatori, p. 173. 
Par ailleurs, l'utilisation de la parataxe pour les constructions relatives semble fréquente dans d'autres variétés de français populaire ${ }^{28}$ et a été également observée en franco-ontarien où, comme dans notre variété, elle semble reliée à la restriction linguistique ${ }^{29}$.

\section{MORPHOLOGIE VERBALE}

Dans ce domaine, nous avons remarqué deux tendances. D'une part, une tendance à la régularisation, vers des formes parfois idiosyncrasiques, d'autre part, une tendance à l'utilisation de formes vernaculaires.

Nous nous limiterons ici à une particularité intéressante du subjonctif, notamment une restriction de son emploi. En effet, à la différence du français standard, le subjonctif n'est jamais utilisé après les expressions de sentiment (ce qui n'est pas sans rappeler l'usage du français préclassique ${ }^{30}$ : c'est honteux qu'eux font des affaires comme f̧a...; elle était si contente qu'on avait pu parler français...

Par ailleurs, l'emploi du subjonctif chez nos locuteurs, restreint aux contextes volitifs, semble révéler une tendance à réduire ce mode à une simple "servitude grammaticale", comme dans d'autres variétés de français hors de France ${ }^{31}$.

En conclusion, le français de Frenchville continue en général et développe les tendances du français populaire. Certains des développements enregistrés (l'effacement du /l/ chez la locutrice moins restreinte, la généralisation de l'auxiliaire avoir dans les verbes non pronominaux) semblent s'expliquer aussi par l'isolement de cette variété, tandis que d'autres (l'effacement du /l/ chez le locuteur plus restreint, eux, l'auxiliaire avoir dans les verbes pronominaux, l'emploi du subjonctif) pourraient renvoyer également aux dialectes d'origine ${ }^{32}$. Finalement, l'empreinte de la restriction

28. Cf. Frei, La grammaire..., p. 182 ; Gadet, Le franfais..., p. 98.

29. Françoise Mougeon, 1976, "Clivage en franco-ontarien", dans Recherches linguistiques à Montréal, 7, p. 163-184. p. 337.

30. Cf. Claude Buridant, 2000, Nouvelle grammaire de l'ancien frangais, Paris, Nathan,

31. Michel Laurier, 1989, "Le subjonctif dans le parler franco-ontarien : un mode en voie de disparition? ", dans Raymond Mougeon et Édouard Beniak (dir.), 1989, Le frangais canadien parlé hors Québec, Sainte-Foy, PUL, p. 105-126 ; Shana Poplack, 1990, « Prescriptions, intuitions et usages : le subjonctif français et la variabilité inhérente ", Langage et société, 54, p. 1-33.

32. Ce qui semble confirmer l'hypothèse de Valdman («L'Acadie...", p. 16) sur l'origine de cette variété. 
linguistique est décelable surtout dans certains aspects de la structure des constructions relatives (la parataxe) et de l'emploi des auxiliaires, et le contact avec l'anglais est particulièrement frappant dans les alternances de code (bien que les phénomènes d'emprunt et de calque soient tout aussi présents que dans d'autres variétés de français en contact avec l'anglais).

ANNEXE

PROFIL LINGUISTIQUE DES LOCUTEURS DU CORPUS DE FRENCHVILLE

\begin{tabular}{|c|c|c|}
\hline & Ll & L2 \\
\hline Sexe & $\mathbf{F}$ & M \\
\hline Relation & Sœur & Frère \\
\hline $\begin{array}{c}\text { Âge au moment } \\
\text { de l'entrevue (1988) }\end{array}$ & 81 ans & 80 ans \\
\hline Lieu de naissance & Frenchville, Pennsylvanie & Frenchville, Pennsylvanie \\
\hline Lieu de résidence & Frenchville, Pennsylvanie & $\begin{array}{c}\text { Frenchville, Monument, } \\
\text { Pennsylvanie (1945-1974), } \\
\text { Frenchville }\end{array}$ \\
\hline Profession & $\begin{array}{l}\text { Fermière et employée } \\
\text { au bureau de poste, } \\
\text { secrétaire de l'école, } \\
\text { puis du comté, } \\
\text { organiste à l'église }\end{array}$ & $\begin{array}{l}\text { Employé itinérant } \\
\text { de la compagnie } \\
\text { Earl Plubell }\end{array}$ \\
\hline $\begin{array}{c}\text { Âge au moment } \\
\text { du décès }\end{array}$ & 85 ans (1992) & 89 ans (1997) \\
\hline $\begin{array}{c}\text { Première } \\
\text { langue apprise }\end{array}$ & Français & Français \\
\hline $\begin{array}{c}\text { Langue } \\
\text { de scolarisation }\end{array}$ & Anglais & Anglais \\
\hline $\begin{array}{l}\text { Langue parlée } \\
\text { à la maison } \\
\text { avec enfants }\end{array}$ & Français & Anglais \\
\hline $\begin{array}{c}\text { Langue parlée } \\
\text { à la maison } \\
\text { avec conjoint }\end{array}$ & Français & Anglais \\
\hline $\begin{array}{l}\text { Langue parlée } \\
\text { entre frère et sœur }\end{array}$ & Français et anglais & $\begin{array}{l}\text { Anglais et un peu } \\
\text { de français }\end{array}$ \\
\hline $\begin{array}{c}\text { Langue parlée } \\
\text { au travail }\end{array}$ & $\begin{array}{l}\text { Anglais. Un peu } \\
\text { de français }\end{array}$ & Anglais \\
\hline
\end{tabular}

